\title{
Perfil metabólico y recomendaciones de entrenamiento físico posterior al confinamiento del COVID-19 en estudiantes de formación de la actividad física y deporte
}

\section{Metabolic profile and recommendations for physical training after confinement of COVID-19 in students of physical activity and sport}

\section{training}

1 Lenin Esteban Loaiza Dávila $\quad$ (iD) https://orcid.org/0000-0002-5769-2795

Universidad Técnica de Ambato, Carrera de Pedagogía de la Actividad Física y Deporte, Tungurahua, Ecuador

e.loaiza@uta.edu.ec

2 Dennis José Hidalgo Alava iD https://orcid.org/0000-0002-2234-5072 Universidad Técnica de Ambato, Carrera de Pedagogía de la Actividad Física y Deporte, Tungurahua, Ecuador djhidalgo@uta.edu.ec

3 Luis Alfredo Jiménez Ruíz https://orcid.org/0000-0001-8704-0563 Universidad Técnica de Ambato, Carrera de Pedagogía de la Actividad Física y Deporte, Tungurahua, Ecuador la.jimenez@uta.edu.ec

4 Jaime Hernández Valdebenito https://orcid.org/0000-0002-5529-7871 Sociedad Chilena para la Educación Física Recreación y Salud, Dirección de Ciencias e Investigación, Santiago de Chile, Chile jhernandez@educacionfisicachile.cl

Artículo de Investigación Científica y Tecnológica Enviado: 24/12/2021

Revisado: 29/12/2021

Aceptado: 03/01/2022

Publicado:08/03/2023

DOI: https://doi.org/10.33262/concienciadigital.v6i1.4.2026

Cítese. Loaiza Dávila, L. E., Hidalgo Alava, D. J., Jiménez Ruíz, L. A., \& Hernández Valdebenito, J. (2023). Perfil metabólico y recomendaciones de entrenamiento físico posterior al confinamiento del COVID-19 en estudiantes de formación de la actividad física y deporte https://doi.org/10.33262/concienciadigital.v6i1.4.2026 
Palabras claves:

perfil

metabólico, grasa corporal, masa muscular, plataforma

metav, entrenamiento físico.
Keywords: metabolic profile, body fat, muscle mass, metav platform, physical training.
Resumen

Introducción: La pandemia del Covid-19 obligo al sistema educativo a adaptar el proceso de enseñanza aprendizaje a una modalidad virtual-telemática, no siendo la formación de los estudiantes universitarios en Pedagogía de la Actividad Física, los cuales a pesar de las características de alto nivel práctico debieron entrar en un estado de inactividad física que altero sus perfiles metabólicos. Objetivo: Determinar el perfil metabólico de los estudiantes en formación de la carrera de Pedagogía de la actividad física y establecer las recomendaciones de entrenamiento físico adecuado, tanto de manera porcentual de sus componentes, así como el tipo de ejercicios adecuados. Metodología: Estudio de enfoque de investigación cuantitativo, a través de un tipo de investigación no experimental por alcance descriptivo y de corte trasversal, desarrollado en una muestra de 460 estudiantes de ambos sexos y de diferentes semestres de formación de la carrera, evaluando su perfil metabólico con la ayuda de la plataforma tecnológica MetaV con respaldo de un análisis estadístico con el paquete SPSS versión 25. Resultados: Se obtuvieron datos de entrada, porcentajes de grasa corporal, masa muscular, masa residual y otros, así como datos de distribución por niveles de parámetros de riesgo metabólico y recomendaciones de entrenamiento físico por perfiles. Conclusión: La investigación permitió determinar que los mayores porcentajes de estudiantes por grupos de sexo se encontraban en el nivel avanzado por entrenamiento físico y en niveles de entrada atlético, deportivo y elite, para los cuales se plantearon recomendaciones de entrenamiento físico arrojados por el sistema MetaV, además de ejercicios físicos diseñados en base a las cadenas cinemáticas y las características biomecánicas de su ejecución.

Abstract

Introduction: The Covid-19 pandemic forced the educational system to adapt the teaching-learning process to a virtual-telematic modality, not being the training of university students in Pedagogy of Physical Activity, which despite the characteristics of high practical level had to enter a state of physical inactivity that altered their metabolic profiles. Objective: To determine the metabolic profile of the students in formation of the Pedagogy of Physical Activity career and to establish the recommendations of adequate 
physical training, both in percentage of its components, as well as the type of adequate exercises. Methodology: A study of quantitative research approach, through a non-experimental type of research by descriptive scope and cross-sectional cut, developed in a sample of 460 students of both sexes and from different semesters of training of the career, evaluating their metabolic profile with the help of the MetaV technological platform supported by a statistical analysis with the SPSS version 25 package. Results: Input data, percentages of body fat, muscle mass, residual mass and others were obtained, as well as distribution data by levels of metabolic risk parameters and physical training recommendations by profiles. Conclusion: The research allowed determining that the highest percentages of students by sex groups were in the advanced level by physical training and in athletic, sports and elite entry levels, for which physical training recommendations were proposed by the MetaV system, in addition to physical exercises designed based on the kinematic chains and the biomechanical characteristics of their execution.

\section{Introducción}

A nivel mundial la situación sanitaria provocada por la pandemia del COVID-19, considerada como una enfermedad de propagación rápida y de fácil contagio, llevo a tomar medidas de distanciamiento social y confinamiento (Villaquiran-Hurtado et al., 2020). El sistema educativo universitario universalmente se vio obligado ante esta situación a adaptarse a un formato virtual-telemático, sin importancia de las características del proceso de enseñanza aprendizaje de las diferentes carreras (PérezLópez \& Cambero-Rivero, 2020).

La formación de los profesionales de la pedagogía de la actividad física y deporte o también conocida a nivel mundial como educación física, está estructurada por un alto componente de asignaturas de desarrollo práctico, el cual está encaminado al desarrollo de las capacidades física y motrices de sus cursantes (Costa et al., 2021), los cuales durante los diferentes años de estudio van desarrollando una condición física y perfil metabólico adecuado (Castro-Jiménez et al., 2018). La falta de la práctica del ejercicio físico, considerando un componente crítico en la formación Almonacid-Fierro et al. (2021), hizo que los docentes busquen alternativas para contrarrestar los efectos de la inactividad y aumento del nivel de sedentarismo, pero a pesar de todos los esfuerzos hubo 
un efecto de perdida de condición física y cambios en la composición corporal que afectaron el perfil metabólico de los estudiantes (Alarcón-Meza \& Hall-López, 2021).

Para Navarrete et al., (2015) el perfil metabólico se determina por el comportamiento de parámetros clínicos y de composición corporal, así como el análisis de la frecuencia de actividad física y hábitos de vida saludable. Existen diferentes métodos para la evaluación del perfil metabólico, basado en la determinación de la alerta metabólica temprana (Chamorro et al., 2018), otros en base a la asociación del índice de masa corporal y relación cintura/estatura con la presión arterial (Diaz \& Development, 2021), análisis antropométricos López et al. (2020), análisis de pliegues cutáneos, perímetros corporales incluido el perímetro del cuello (Pedreros et al., 2018), métodos de bioempedancia (Dopsaj et al., 2021), entre otros.

En relación con la composición corporal, es importante recalcar que el análisis del índice de masa corporal (IMC), relación del perímetro cintura (PC) y el porcentaje de masa grasa corporal (\%MG), son métodos accesibles y de bajo costo, utilizados en estudios investigativos de epidemiología y salud pública. Sea cual sea el método utilizado, estos arrojan una serie de datos cuantitativos que permiten clasificar a los sujetos evaluados en diferentes niveles de riesgo metabólico, basados en el sobrepeso o niveles de obesidad (Espinoza-Navarro et al., 2020), o al contario la deficiencia de peso corporal y un porcentaje disminuido de masa muscular (Buitrago et al., 2021), determinando de esta manera los dos lados opuestos del perfil metabólico.

El perfil metabólico que determina un riesgo, se caracteriza por los problemas de sobrepeso y obesidad que son el resultado de la formación de grasa corporal anormal o excesiva que puede ser perjudicial para la salud (Villena, 2018). El método más adecuado para determinar los niveles de estos 2 parámetros es el índice de masa corporal (IMC), que no es más que es una relación simple entre el peso corporal y la altura (Massaroli et al., 2021). El índice se calcula como la relación entre el peso corporal en kilogramos y la altura en metros al cuadrado $(\mathrm{kg} / \mathrm{m} 2)$.

Según la OMS, el diagnóstico de sobrepeso u obesidad en adultos se establece en los siguientes casos:

- $\quad$ IMC mayor o igual a 25 - sobrepeso;

- IMC mayor o igual a 30 - obesidad.

El IMC es la medida más conveniente para evaluar el nivel de obesidad y sobrepeso en una población determinada, ya que es el mismo para su evaluación en ambos sexos y para todos los grupos etarios en la adultez. Sin embargo, se debe considerar que el IMC un criterio aproximado, ya que puede corresponder a diferentes grados de completitud en diferentes personas o poblaciones (Chavarría et al., 2017). 
Abordando las causas que producen la obesidad y sobrepeso, determinamos la principal como el desequilibrio energético de ingesta alimenticia, es decir que el contenido calórico de la dieta diaria excede los requerimientos energéticos que el cuerpo requiere, producto de un mayor consumo de alimentos con alta densidad energética y alto contenido de grasas (Velasco et al., 2018). La segunda causa es la disminución de la actividad o entrenamiento físicos debido al carácter cada vez más sedentario de muchas actividades y los cambios en la transportación que cada vez es menos activo. Los diferentes cambios en la dieta y la actividad física a menudo son el resultado de cambios de carácter ambiental y social (Huaman \& Bolaños, 2020).

Los niveles altos del perfil metabólico determinan a sujetos con un peso corporal adecuado, un porcentaje de grasa reducido y un mayor porcentaje de masa muscular, son sujetos que implementa sistemas de entrenamiento para mantener niveles adecuados de masa muscular, siendo esto un factor de prevención de enfermedades por los niveles de fuerza que estos presentan y la función metabólica del tejido muscular y cardiaco (Hernández \& Domínguez, 2019).

Según Ceballos-Gurrola et al. (2020), el ejercicio físico y una buena nutrición son parte de un conjunto de parámetros que se deben seguir al momento de generar cambios en el perfil metabólico del ser humano a corto plazo. El mecanismo por el cual el ejercicio tiene un efecto beneficioso sobre el metabolismo de los músculos y de todo el cuerpo, quizás, es la participación en la regulación de los procesos catabólicos y anabólicos en función de los requerimientos energéticos (Hernández \& Licea-Puig, 2017), además de mejorar la función mitocondrial activando la biogénesis y remodelación mitocondrial, aumentando la densidad de las mitocondrias y su vitalidad (Mahecha, 2021).

En concordancia con las normas disponibles para el entrenamiento físico, direccionadas hacia los trastornos metabólicos de sobrepeso o bajo peso, la regularidad y la intensidad moderada son los principios fundamentales del proceso de ejercitación (Fuentes-Barria et al., 2021). Diferentes autores recomiendan un mínimo de 45 minutos entrenamiento físico de intensidad de carácter moderada por día para mantener una condición física y composición corporal adecuada (Tapia-Serrano et al., 2020). Sin embargo, se da preferencia a caminar durante 60 minutos de intensidad moderada en combinación con otros tipos de actividad direccionadas a la disminución del tejido graso por la incursión del sistema energético aeróbico (Silva, 2018). Es necesario incluir períodos de actividad física de 10 a 15 minutos con o sin equipo de ejercicio simple, y evitar sentarse durante el tiempo libre. Una actividad física de 45 minutos lograda durante 3 sesiones de 15 minutos por día equivale a un gasto energético de $1500 \mathrm{kcal}-1800 \mathrm{kcal}$ por semana (Cruz et al., 2020). La expansión del entrenamiento físico, tanto en intensidad como en duración, debe ser gradual (en 15 min / sesión / semana), comenzando con ejercicio de baja intensidad es decir menores a 3 MET de trabajo (Pérez et al., 2019). 
Además se considera la combinación óptima de ejercicio anaeróbico o de trabajo muscular y aeróbico, que le permite lograr una reducción más significativa del peso corporal y la masa grasa, mientras se mantiene la masa magra en comparación con el ejercicio aeróbico o anaeróbico aislado (Aguilar et al., 2014). De igual manera, el ejercicio regular aumenta la sensibilidad a la insulina, los niveles de triglicéridos y la morbilidad y mortalidad cardiovascular dentro de los procesos fisiológicos.

De esta manera el entrenamiento físico regular, estructurados en base a ejercicios aeróbicos y anaeróbicos, debe ser un componente integral de la prevención y el tratamiento de los trastornos metabólicos, incluido el síndrome metabólico (MantillaMorrón et al., 2018).

Tomando en cuenta la problemática analizada y los problemas conocidos por falta la práctica de la actividad física de los estudiantes universitarios de formación en Pedagogía de la Actividad Física como producto de la reducción del entrenamiento físico habitual en el desarrollo del proceso de enseñanza aprendizaje, se plantea como objetivo principal del estudio determinar el perfil metabólico de los estudiantes en formación de la carrera de Pedagogía de la actividad física y establecer las recomendaciones de entrenamiento físico adecuado, tanto de manera porcentual de sus componentes así como el tipo de ejercicios adecuados.

\section{Metodología}

El estudio se planteó en base a un enfoque de investigación cuantitativo, a través de un tipo de investigación no experimental por alcance descriptivo y de corte trasversal, aplicando los métodos analítico y deductivo para el desarrollo de la investigación desde lo teórico a lo práctico.

La población de estudio estuvo representada por estudiantes de la carrera de Pedagogía de la Actividad Física y Deporte de la Universidad Técnica de Ambato - Ecuador. A través de un muestreo probabilístico se determinó una muestra de 460 estudiantes de ambos sexos y de diferentes semestres de formación de la carrera, los cuales tuvieron las siguientes características (tabla 1).

\section{Tabla 1}

Caracterización de la muestra de estudio

\begin{tabular}{|c|c|c|c|c|c|c|c|}
\hline \multirow{2}{*}{$\begin{array}{c}\text { Variables de } \\
\text { caracterización }\end{array}$} & \multicolumn{2}{|c|}{$\begin{array}{c}\text { Masculino } \\
\text { (n } 326-70.9 \%)\end{array}$} & \multicolumn{2}{|c|}{$\begin{array}{c}\text { Femenino } \\
\text { (n } 134-29.1 \%)\end{array}$} & \multirow[t]{2}{*}{$\mathrm{P}$} & \multicolumn{2}{|c|}{$\begin{array}{c}\text { Total } \\
\text { (n } 460-100 \%)\end{array}$} \\
\hline & M & $\mathrm{DS}$ & M & DS & & M & DS \\
\hline Edad cronológica & 23.5 & 5.2 & 21.7 & 2.1 & 0.000 & 22.9 & 4.6 \\
\hline
\end{tabular}


Tabla 1

Caracterización de la muestra de estudio (continuación)

\begin{tabular}{cccccccc}
\hline \multirow{2}{*}{$\begin{array}{c}\text { Variables de } \\
\text { caracterización }\end{array}$} & \multicolumn{2}{c}{ Masculino } & \multicolumn{2}{c}{ Femenino } & \multicolumn{3}{c}{ Total } \\
& M $326-70.9 \%)$ & \multicolumn{2}{c}{ (n 134-29.1\%) } & P & \multicolumn{2}{c}{ (n 460-100\%) } \\
\hline Peso corporal & 69.2 & 12.9 & 57.8 & 14.7 & 0.000 & 65.9 & 14.4 \\
Estatura & 169.4 & 6.5 & 156.5 & 5.7 & 0.000 & 165.6 & 8.6 \\
IMC & 24.1 & 4.3 & 23.6 & 5.7 & 0.009 & 23.9 & 4.8
\end{tabular}

Nota. Análisis descriptivo de valores medios (M) y desviaciones estándares (DS) con diferencias significativas en un nivel de $\mathrm{P}<0.05$; elaboración propia de los autores

Fuente: Elaboración propia basado en datos arrojados por el sistema MetaV

La muestra de estudio en su mayoría estuvo representada por estudiantes del sexo masculino, en relación con las variables de edad cronológica, peso corporal, estatura e IMC, los valores medios de este grupo descriptivamente fueron mayores que los del grupo femenino, resultados que estadísticamente se respalda con un nivel de significación de $\mathrm{P}$ $<0.05$ en todas las variables. Por la existencia de diferencias significativas todos los resultados de evaluación del perfil metabólico se presentan segmentados por sexo y de manera general.

La técnica aplicada fue la observación y como instrumento la plataforma tecnológica de aplicación online de MetaV creada por la Sociedad Chilena de Educación Física Recreación y Salud -SCHEFRES, la cual permite realizar la evaluación de múltiples parámetros al objeto de determinar la real valoración del estado de alerta metabólica temprana y de esta manera el perfil metabólico del evaluado.

La plataforma desarrolla su análisis a través del ingreso de datos fisiológicos (edad, peso corporal, estatura), medidas antropométricas (perímetro de la cintura, cadera, brazo derecho relajado, pierna derecha medial y cuello), frecuencia de actividad física por tiempo y su periodización semanal, hábitos de vida saludable (consumo de tabaco y alcohol) y valores sistólicos y diastólicos de la presión arterial. Estos datos de entrada permiten de manera automatizada calcular parámetros de IMC, porcentaje de grasa corporal, masa muscular y porcentaje de masa residual y otros, fidelizados a través de logaritmos basados en formulas internacionales (tabla 2) y el modelo antropométrico de Heat-Carter que es una descripción cuantificada de la forma física, que se expresa a través de una escala numérica y gráfica, con el objetivo de conciliar universalmente estos protocolos. 
Tabla 2

Formulas del sistema automatizado de la plataforma MetaV

\begin{tabular}{|c|c|c|}
\hline Variable & Masculino & Femenino \\
\hline IMC & \multicolumn{2}{|c|}{ peso $[\mathrm{kg}] /$ estatura [m2] } \\
\hline $\begin{array}{l}\text { Porcentaje de } \\
\text { grasa }\end{array}$ & $\begin{array}{c}\text { Densidad Corporal }(\mathrm{DC})=\mathrm{C}-[\mathrm{M} * \mathrm{Log} \\
\quad(\text { Suma Pliegues*0,4) }] \\
\% \mathrm{G}=(4,95 / \mathrm{DC}-4,5) * 100\end{array}$ & $\begin{array}{c}\text { Densidad Corporal }(\mathrm{DC})=\mathrm{C}-[\mathrm{M} * \\
\log (\text { Suma Pliegues } * 0,4)] \\
\% \mathrm{G}=(4,95 / \mathrm{DC}-4,5) * 100\end{array}$ \\
\hline $\begin{array}{l}\text { Porcentaje Masa } \\
\text { Muscular }\end{array}$ & $\begin{array}{c}\text { Ecuación de Drinkwater y Ross }(7) \\
\mathrm{MM}=(\mathrm{Z} \times 2,99+25,55) /(170,18 / \mathrm{T}) 3\end{array}$ & $\begin{array}{l}\text { Ecuación de Drinkwater y Ross (7) } \\
\qquad \begin{array}{c}\mathrm{MM}=(\mathrm{Z} \times 2,99+25,55) \\
/(170,18 / \mathrm{T}) 3\end{array}\end{array}$ \\
\hline $\begin{array}{l}\text { Porcentaje masa } \\
\text { residual }\end{array}$ & Peso corporal $* 24,1 / 100$ & Peso corporal $* 20,9 / 100$ \\
\hline
\end{tabular}

Fuente: Elaboración propia basado en logaritmos sistema MetaV

La plataforma de igual manera permite determinar diferentes niveles en base a un análisis del perímetro de cintura, grasa corporal y masa muscular, esto basado en que los parámetros de las ecuaciones antropométricas hacen posible adaptarse asertivamente en un 99,5\% a toda la población mundial, ya que poseen derivación de desviación estándar para todas las etnias y grupos etarios sobre los 15 años de edad, si bien el estudio de población especial hecho en Chile con una muestra de 7575 personas tiene el matiz somático de ese país los estándares ocupados son intencionalmente validados por la ISAK.

El sistema MetaV como informe final permite categorizar al evaluado en base a todos los datos analizados, en 9 niveles de perfil metabólico asociados a diferentes colores (figura 1), caracterizados por el nivel de entrada metabólica, un scoring arrojado por el propio sistema MetaV y el nivel de la clase en base a sus hábitos de actividad física.

\section{Figura 1}

Niveles del perfil metabólico según la plataforma MetaV

\begin{tabular}{|ccc|}
\hline Nivel de Entrada & Scoring & Nivel de la Clase \\
\hline Alto Riesgo & $3.6-7$ & Nivel Básico \\
\hline Riesgo & $2.6-3.5$ & Nivel Básico \\
\hline Sedentario & $1.1-2.5$ & Nivel Básico \\
\hline Normal Sedentario & $0.3-1$ & Nivel Intermedio \\
\hline Normal & $-0.5-0.2$ & Nivel Intermedio \\
\hline En forma & $-1.5--0.6$ & Nivel Intermedio \\
\hline Deportivo & $-2.9--1.6$ & Nivel Avanzado \\
\hline Atlético & $-3.9--3$ & Nivel Avanzado \\
\hline Elite & $-5--4$ & Nivel Avanzado \\
\hline
\end{tabular}

Fuente: Scoring automático de la plataforma MetaV 
En base al informe de perfil metabólico individual del evaluado y su nivel asignado por la plataforma MetaV, el sistema determina recomendaciones de entrenamiento físico basado en el objetivo de nivelación de los parámetros de composición corporal, la distribución en componentes de tiempo de calentamiento, trabajo aeróbico y de trabajo muscular en base a la disponibilidad de tiempo del evaluado para su ejecución.

Se aplicó el paquete estadístico SPSS 27 para el sistema IBM, realizando un análisis de distribución frecuencial y porcentual en las diferentes variables cualitativas y un análisis descriptivo de valores medios y desviaciones estándares para variables cuantitativas. De igual manera se realizó la prueba de normalidad de Kolmogorov-Smirnov para todas las variables cuantitativas determinando la aplicación de la prueba no paramétrica $U$ de Mann-Withney para muestras independientes con distribución anormal, así como la prueba $\mathrm{H}$ de Kruskal-Wallis para muestras múltiples como es el caso de los niveles de perfil metabólico MetaV para determinar la diferencia entre las recomendaciones de los componentes del entrenamiento físico.

\section{Resultados}

Además de los parámetros básicos de edad, peso corporal, estatura, hábitos de vida saludable y presión arterial con su componente sistólico y diastólico, se ingresaron los diferentes perímetros corporales, basados en los protocolos básico ISAK, diferenciándolos por grupos de sexo (tabla 3).

Tabla 3

Perímetros corporales por grupos de sexo

\begin{tabular}{|c|c|c|c|c|c|c|c|}
\hline \multirow[t]{2}{*}{ Perímetros corporales } & \multicolumn{2}{|c|}{$\begin{array}{c}\text { Masculino } \\
\text { (n 326) }\end{array}$} & \multicolumn{2}{|c|}{$\begin{array}{c}\text { Femenino } \\
\text { (n 134) }\end{array}$} & \multirow[t]{2}{*}{$\mathrm{P}$} & \multicolumn{2}{|c|}{$\begin{array}{c}\text { Total } \\
(\mathrm{n} 460)\end{array}$} \\
\hline & $\mathrm{M}$ & DS & $\mathrm{M}$ & DS & & $\mathrm{M}$ & DS \\
\hline Perímetro cintura & 85.7 & 10.5 & 76.7 & 7.4 & 0.000 & 83.1 & 10.5 \\
\hline Perímetro cadera & 94.5 & 8.9 & 92.2 & 8.9 & 0.012 & 93.8 & 9.1 \\
\hline Perímetro brazo derecho & 29.9 & 4.5 & 26.7 & 5.4 & 0.000 & 28.9 & 4.9 \\
\hline Perímetro pierna derecha & 52.9 & 7.8 & 50.8 & 6.5 & 0.010 & 52.3 & 7.5 \\
\hline Perímetro cuello & 38.1 & 3.1 & 33.6 & 2.7 & 0.000 & 36.8 & 3.6 \\
\hline
\end{tabular}

Los perímetros ingresados presentaron diferencias significativas entre los grupos por sexo en un nivel de $\mathrm{P}<0.05 \mathrm{y}$ en todos los casos por las características del sexo masculino los valores medios fueron superiores a los del sexo femenino. Estos perímetros y demás parámetros de entrada, procesados automatizadamente por el sistema MetaV, permitieron obtener los parámetros de porcentajes de composición corporal de igual manera segmentados en grupos por sexo (tabla 4). 


\section{Tabla 4}

Porcentajes de parámetros de composición corporal por grupos de sexo.

\begin{tabular}{|c|c|c|c|c|c|c|c|}
\hline \multirow[t]{2}{*}{ Perímetros corporales } & \multicolumn{2}{|c|}{$\begin{array}{l}\text { Masculino } \\
\text { (n 326) }\end{array}$} & \multicolumn{2}{|c|}{$\begin{array}{l}\text { Femenino } \\
\text { (n 134) }\end{array}$} & \multirow[t]{2}{*}{$\mathrm{P}$} & \multicolumn{2}{|c|}{$\begin{array}{c}\text { Total } \\
(\mathrm{n} 460)\end{array}$} \\
\hline & M & DS & $\mathrm{M}$ & DS & & $\mathrm{M}$ & DS \\
\hline Porcentaje de grasa $(\%)$ & 18.4 & 6.3 & 28.1 & 6.8 & 0.000 & 21.2 & 7.7 \\
\hline $\begin{array}{c}\text { Porcentaje de masa muscular } \\
\qquad(\%)\end{array}$ & 48.5 & 6.3 & 43.4 & 6.9 & 0.000 & 47.1 & 6.9 \\
\hline $\begin{array}{c}\text { Porcentaje de masa residual y } \\
\text { otros }(\%)\end{array}$ & 33.1 & 1.4 & 28.5 & 1.6 & 0.000 & 31.7 & 2.5 \\
\hline
\end{tabular}

Fuente: Elaboración propia basado en datos arrojados por el sistema MetaV

Los nuevos parámetros obtenidos permitieron determinar que, en relación con el porcentaje de grasa, el sexo femenino presento un valor medio mayor en $10.3 \%$ que el grupo de sexo masculino, en el porcentaje de masa muscular el sexo masculino fue mayor en un $5.1 \%$ y en el porcentaje de masa residual y otros fue mayor en $4.6 \%$. estadísticamente existieron diferencias significativas en un nivel de $\mathrm{P}<0.05$ en todos los porcentajes analizados.

Los valores porcentuales obtenidos permitieron en base a los baremos propios del sistema, caracterizar a las muestras de estudio por sexo en diferentes niveles que determinan la existencia de un parámetro adecuado o en situación de riesgo, empezando por el parámetro del perímetro de la cintura (tabla 5).

\section{Tabla 5}

Distribución por niveles en base al perímetro de la cintura

\begin{tabular}{ccccccc}
\hline & \multicolumn{2}{c}{ Masculino } & \multicolumn{2}{c}{ Femenino } & \multicolumn{2}{c}{ Total } \\
\cline { 2 - 7 } Nivel & $\mathrm{f}$ & $\%$ & $\mathrm{f}$ & $\%$ & $\mathrm{f}$ & $\%$ \\
\hline $\begin{array}{c}\text { Aceptable } \\
\text { Sobre lo } \\
\text { normal }\end{array}$ & 231 & 70.9 & 127 & 94.8 & 77.8 & 77.8 \\
Riesgo & 50 & 15.3 & 1 & 0.7 & 11.1 & 11.1 \\
\hline Total & 326 & 100.0 & 134 & 100.0 & 100.0 & 100.0 \\
\hline
\end{tabular}

Fuente: Elaboración propia basado en datos arrojados por el sistema MetaV

A pesar del estado de inactividad física que los estudiantes a travesaron por las medidas adoptadas en la pandemia del COVID-19, el mayor porcentaje de la muestra de estudio tanto en el sexo masculino y con mayor valor en el sexo femenino, el perímetro de la cintura se distribuyó en un nivel aceptable, no obstante, un porcentaje significativo (15.3) de estudiantes del sexo masculino presentaron un nivel de riesgo. 
De igual manera observamos la distribución de niveles en relación con el porcentaje de grasa corporal (tabla 6).

\section{Tabla 6}

Distribución por niveles en base al porcentaje de grasa corporal

\begin{tabular}{ccccccr}
\hline \multirow{2}{*}{ Nivel } & \multicolumn{2}{c}{ Masculino } & \multicolumn{2}{c}{ Femenino } & \multicolumn{2}{c}{ Total } \\
\cline { 2 - 7 } & $\mathrm{f}$ & $\%$ & $\mathrm{f}$ & $\%$ & $\mathrm{f}$ & $\%$ \\
\hline Riesgo & 33 & 10.1 & 0 & 0 & 33 & 7.2 \\
Aceptable & 40 & 12.3 & 3 & 2.2 & 43 & 9.3 \\
En forma & 43 & 13.2 & 4 & 3.0 & 47 & 10.2 \\
Élite & 210 & 64.4 & 127 & 94.8 & 337 & 73.3 \\
\hline Total & 326 & 100.0 & 134 & 100.0 & 460 & 100.0
\end{tabular}

Fuente: Elaboración propia basado en datos arrojados por el sistema MetaV

En relación con este parámetro en ambos grupos el mayor porcentaje y sobre todo en el sexo femenino, se encontraron en un nivel élite según el porcentaje de grasa corporal, evidenciando además que solo en el grupo de sexo masculino existo un porcentaje correspondiente a 1/10 parte de la muestra en un nivel de riesgo.

Como último dato de distribución por niveles se observaron los resultados en base al porcentaje de masa muscular (tabla 7).

Tabla 7

Distribución por niveles en base al porcentaje de masa muscular

\begin{tabular}{|c|c|c|c|c|c|c|}
\hline \multirow{2}{*}{ Nivel } & \multicolumn{2}{|c|}{ Masculino } & \multicolumn{2}{|c|}{ Femenino } & \multicolumn{2}{|c|}{ Total } \\
\hline & $\mathrm{f}$ & $\%$ & $\mathrm{f}$ & $\%$ & $\mathrm{f}$ & $\%$ \\
\hline Aceptable & 35 & 10.7 & 40 & 29.9 & 75 & 16.3 \\
\hline Normal & 36 & 11.0 & 35 & 26.1 & 71 & 15.4 \\
\hline En forma & 60 & 18.4 & 18 & 13.4 & 78 & 17.0 \\
\hline Élite & 195 & 59.8 & 41 & 30.6 & 236 & 51.3 \\
\hline Total & 326 & 100.0 & 134 & 100.0 & 460 & 100.0 \\
\hline
\end{tabular}

Fuente: Elaboración propia basado en datos arrojados por el sistema MetaV

De igual manera por las características y memoria física de las muestras de estudio, la distribución en ambos grupos por sexo con una mayor diferencia en el sexo masculino fue en el nivel élite de porcentaje de masa muscular, evidenciando que en relación con este parámetro no se observaron niveles de riesgo con valores dentro del nivel aceptable.

En base a todos los datos de entrada, así como los procesados automatizadamente por la plataforma MetaV, se pudieron obtener los diferentes niveles de perfil metabólico 
específicos del sistema, construidos por el nivel de composición corporal y la frecuencia de actividad física (tabla 8).

\section{Tabla 8}

Distribución por niveles de perfil metabólico de la plataforma MetaV en base a parámetros de composición corporal y frecuencia de actividad física

\begin{tabular}{ccccccc}
\hline \multirow{2}{*}{ Niveles plataforma MetaV } & \multicolumn{2}{c}{ Masculino } & \multicolumn{2}{c}{ Femenino } & \multicolumn{2}{c}{ Total } \\
\cline { 2 - 7 } & $\mathrm{f}$ & $\%$ & $\mathrm{f}$ & $\%$ & $\mathrm{f}$ & $\%$ \\
\hline Alto Riesgo/Nivel Básico & 4 & 1.2 & 5 & 3.7 & 9 & 2.0 \\
Riesgo/Nivel Básico & 15 & 4.6 & 6 & 4.5 & 21 & 4.6 \\
Sedentario/Nivel Básico & 33 & 10.1 & 9 & 6.7 & 42 & 9.1 \\
\hline Normal Sedentario/Nivel & 23 & 7.1 & 14 & 10.4 & 37 & 8.0 \\
Intermedio & 33 & 10.1 & 12 & 9.0 & 45 & 9.8 \\
Normal/Nivel Intermedio & 47 & 14.4 & 22 & 16.4 & 69 & 15.0 \\
En Forma/Nivel Intermedio & 76 & 23.3 & 31 & 23.1 & 107 & 23.3 \\
\hline Deportivo/Nivel Avanzado & 42 & 12.9 & 13 & 9.7 & 55 & 12.0 \\
Atlético Nivel Avanzado & 53 & 16.3 & 22 & 16.4 & 75 & 16.3 \\
Elite/Nivel Avanzado & 326 & 100.0 & 134 & 100.0 & 460 & 100.0 \\
Total & & & & & \\
\end{tabular}

Fuente: Elaboración propia basado en datos arrojados por el sistema MetaV

La distribución en base al sistema MetaV determino que los mayores porcentajes en ambos grupos por sexo se encontraron en un nivel Deportivo/Nivel avanzado de entrenamiento y con menor frecuencia en un nivel Elite/Nivel avanzado de entrenamiento, sin embargo, un $16.9 \%$ del grupo de sexo masculino se encontró en niveles de Alto riesgo a Sedentario con un nivel básico de entrenamiento, de igual manera un $14.9 \%$ del grupo de sexo femenino.

Tomando en cuenta los niveles de perfil metabólico MetaV, los parámetros de composición corporal y las recomendaciones internacionales sobre entrenamiento físico, el sistema arrojo las recomendaciones de entrenamiento por componentes de trabajo (tabla 9).

\section{Tabla 9}

Recomendaciones de entrenamiento físico por perfil metabólico

\begin{tabular}{ccccccc}
\hline Perfil metabólico MetaV & $\begin{array}{c}\text { Componentes } \\
\text { entrenamiento }\end{array}$ & $\mathrm{n}$ & $\begin{array}{c}\text { \% x } \\
\text { componente }\end{array}$ & $\begin{array}{c}\text { Min x } \\
45 \mathrm{~min}\end{array}$ & $\begin{array}{c}\text { Min x } \\
60 \mathrm{~min}\end{array}$ & $\begin{array}{c}\text { Min x } \\
90 \mathrm{~min}\end{array}$ \\
\hline $\begin{array}{c}\text { Alto Riesgo/Nivel Básico } \\
\text { Riesgo/Nivel Básico }\end{array}$ & \% calentamiento & & 10 & 5 & 6 & 9 \\
$\begin{array}{c}\text { \% trabajo aeróbico } \\
\text { Sedentario/Nivel Básico }\end{array}$ & 72 & 70 & 32 & 42 & 63 \\
\hline \multicolumn{2}{c}{ Total trabajo muscular } & & 20 & 9 & 12 & 18 \\
\hline
\end{tabular}


Tabla 9

Recomendaciones de entrenamiento físico por perfil metabólico (continuación)

\begin{tabular}{|c|c|c|c|c|c|c|}
\hline Perfil metabólico MetaV & $\begin{array}{l}\text { Componentes } \\
\text { entrenamiento }\end{array}$ & $\mathrm{n}$ & $\begin{array}{c}\% \mathrm{x} \\
\text { componente }\end{array}$ & $\begin{array}{c}\operatorname{Min} \times 45 \\
\min \end{array}$ & $\begin{array}{c}\operatorname{Min} \times 60 \\
\min \end{array}$ & $\begin{array}{c}\operatorname{Min} x 90 \\
\min \end{array}$ \\
\hline \multirow{3}{*}{$\begin{array}{l}\text { Normal Sedentario/Nivel } \\
\text { Intermedio Normal/Nivel } \\
\text { Intermedio En Forma/Nivel } \\
\text { Intermedio }\end{array}$} & $\%$ calentamiento & \multirow{3}{*}{151} & 5 & 2 & 3 & 5 \\
\hline & $\%$ trabajo aeróbico & & 50 & 23 & 30 & 45 \\
\hline & $\%$ trabajo muscular & & 45 & 20 & 27 & 41 \\
\hline \multicolumn{3}{|c|}{ Total } & 100 & 45 & 60 & 90 \\
\hline \multirow{3}{*}{$\begin{array}{l}\text { Deportivo/Nivel Avanzado } \\
\text { Atlético Nivel Avanzado } \\
\text { Elite/Nivel Avanzado }\end{array}$} & $\%$ calentamiento & \multirow{3}{*}{237} & 5 & 2 & 3 & 5 \\
\hline & $\%$ trabajo aeróbico & & 30 & 14 & 18 & 27 \\
\hline & $\%$ trabajo muscular & & 65 & 29 & 39 & 59 \\
\hline \multicolumn{3}{|c|}{ Total } & 100 & 45 & 60 & 90 \\
\hline
\end{tabular}

Fuente: Elaboración propia basado en datos arrojados por el sistema MetaV

Las recomendaciones se construyeron en base a la necesidad de trabajo aeróbico y muscular, según el perfil metabólico de cada sujeto evaluado, observando de manera gradual la distribución de porcentajes por componente y el aumento progresivo de la temporalidad del entrenamiento. Para evidenciar que las recomendaciones son diferentes en base a cada perfil determinado, se aplicó un análisis estadístico (tabla 10), que permitió determinar las diferencias significativas entre las sugerencias planteadas en la muestra de estudio.

\section{Tabla 10}

Análisis de diferencias estadísticas entre las recomendaciones de componentes de entrenamiento por perfil metabólico MetaV

\begin{tabular}{cccc}
\hline Estadísticos & \% calentamiento & \% trabajo aeróbico & \% trabajo de musculación \\
\hline H de Kruskal-Wallis & 437.233 & 456.541 & 456.541 \\
$\mathrm{gl}$ & 2 & 2 & 2 \\
\hline Sig. asintótica & 0.000 & 0.000 & 0.000 \\
\hline
\end{tabular}

Fuente: Elaboración propia basado en datos arrojados por el sistema MetaV y procesados en el paquete estadístico SPSS

La prueba estadística aplicada para comparación de múltiples grupos arrojo que en los 3 componentes analizados existían diferencias significativas en un nivel de $\mathrm{P}<0.05$, que determina que para cada perfil existen diferentes porcentajes y valores temporales de calentamiento, trabajo aeróbico y trabajo de musculación.

Como aporte de la investigación realizada y en respaldo a las recomendaciones por componentes de entrenamiento se planteó recomendaciones de ejercicios físicos por nivel de perfil metabólico en base al nivel de entrada y nivel de entrenamiento (figura 2). 


\section{Figura 2}

Ejercicios físicos por perfil metabólico según niveles de entrada y entrenamiento

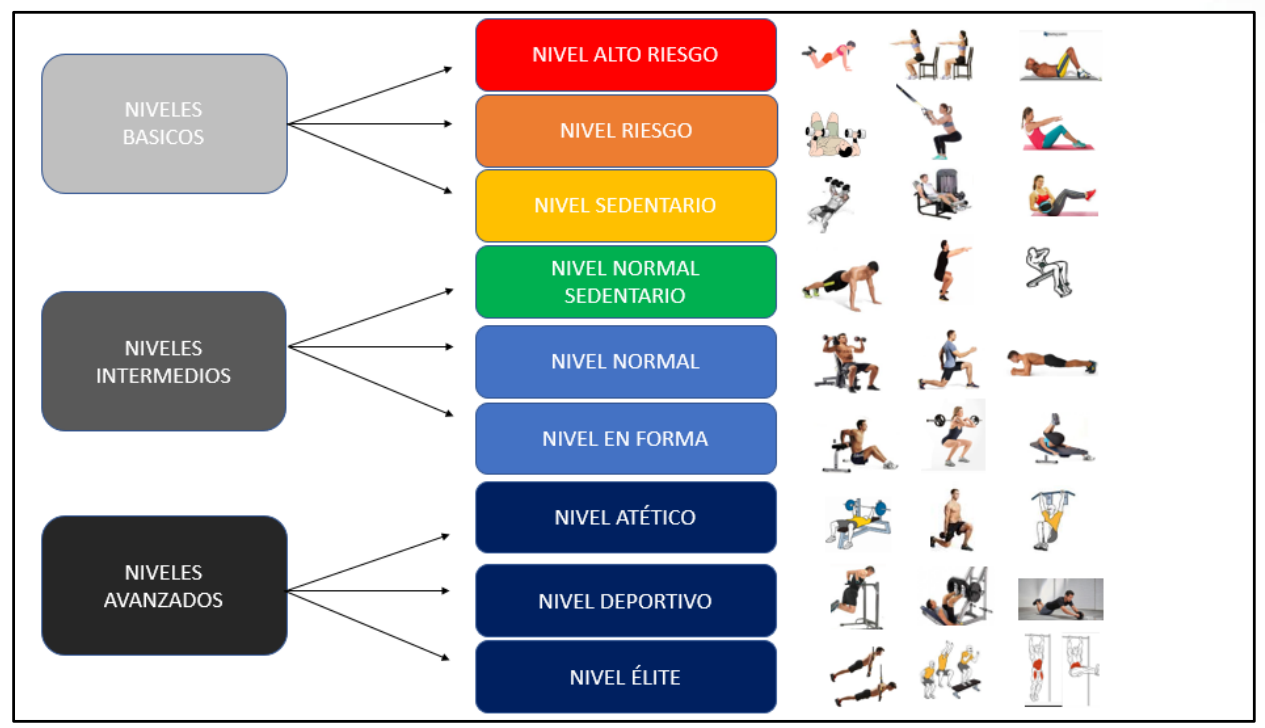

Los tipos de ejercicios planteados fueron totalizadores, de transferencia, musculares de empuje, multiarticulares sin utilización de sobrecarga, diseñados en base a las cadenas cinemáticas y las características biomecánicas de su ejecución, con recomendaciones de dosificación de repeticiones, frecuencia de series y descansos activos entre series y repeticiones, y sobre todo en dependencia de las posibilidades que el evaluado presenta según su perfil metabólico.

\section{Conclusiones}

- A través de la plataforma MetaV y su sistema automatizado de determinación de perfiles metabólicos en base a la composición corporal, frecuencia de entrenamiento y hábitos de vida saludable, se determinó que la muestra de estudio de estudiantes de la carrera de Pedagogía de la actividad Física y Deporte se encontraban en niveles elites y aceptables de porcentajes de grasa corporal, masa muscular y perímetro de la cintura, observando que el porcentaje de estudiantes en riesgo metabólico era reducido.

- El análisis de los diferentes parámetros de entrada y procesados por el sistema MetaV, permitió categorizar a los estudiantes en diferentes niveles de perfiles metabólico, observando que los mayores porcentajes de estudiantes por grupos de sexo se encontraban en el nivel avanzado por entrenamiento físico y en niveles de entrada atlético, deportivo y elite, fenómeno que se da por las características de estos estudiantes y la memoria física que el organismo humano sostiene a pesar 
de la inactividad física por aislamiento obligatorio por la pandemia del COVID19.

- Las recomendaciones de entrenamiento físico se realizaron en base a los datos arrojados por el sistema MetaV, distribuyendo en base al perfil metabólico del evaluado y la necesidad de trabajo aeróbico y muscular con un aumento de manera gradual de la distribución de los porcentajes por componente y el aumento progresivo de la temporalidad del entrenamiento, además de ejercicios físicos como totalizadores, de transferencia, musculares de empuje, multiarticulares sin utilización de sobrecarga, diseñados en base a las cadenas cinemáticas y las características biomecánicas de su ejecución, con recomendaciones de dosificación de repeticiones, frecuencia de series y descansos activos entre series y repeticiones, y sobre todo en dependencia de las posibilidades que el evaluado presenta.

\section{Agradecimientos}

Los autores desean agradecer a la Universidad Técnica de Ambato (UTA) y a la Dirección de Investigación y Desarrollo (DIDE) por su apoyo para la exitosa ejecución de este trabajo a través del proyecto de investigación titulado "PERFIL GENÉTICO COMO DETERMINANTE DE LA SALUD Y EL RIESGO METABÓLICO EN ESTUDIANTES universitarios tras un aislamiento domiciliario", código PFCHE17. De igual manera a la Sociedad Chilena de Educación Física Recreación y Salud-SCHEFRES que a través de la empresa MetaV se proporcionó la accesibilidad de los créditos de evaluación necesarios para el desarrollo de la investigación.

\section{Referencias bibliográficas}

Aguilar Cordero, M. J., Ortegón Piñero, A., Mur Villar, N., Sánchez García, J. C., García Verazaluce, J. J., García, I. G., \& Sánchez López, A. M. (2014). Programas de actividad física para reducir sobrepeso y obesidad en niños y adolescentes; revisión sistemática. Nutrición Hospitalaria, 30(4), 727-740. https://doi.org/10.3305/nh.2014.30.4.7680

Alarcón-Meza, E. I., \& Hall-López, J. A. (2021). Actividad física en estudiantes deportistas universitarios, previo y en el confinamiento por pandemia asociada al COVID-19. Retos, 2041(39), 572-575.

Almonacid-Fierro, A., Vargas Vitoria, R., Mondaca Urrutia, J., \& Sepúlveda-Vallejos, S. (2021). Prácticas profesionales en tiempos de pandemia Covid-19: Desafíos para la formación inicial en profesorado de Educación Física (Professional practices intimes 
of Covid-19 pandemic: Challenges for Physical Education initial teaching training). Retos, 42, 162-171. https://doi.org/10.47197/retos.v42i0.87353

Buitrago, R. A., Lobach, Y., \& Portillo, I. (2021). Musculación y los nuevos valores estéticos en la narrativa social hispana contemporánea. 25-52. https://doi.org/10.24310/JPEHMjpehmjpehm.v3i213126

Castro-Jiménez, L. E., Argüello-Gutiérrez, Y. P., Vásquez, N., Valderrama, J., Tovar, H., Godoy, O., \& Sabogal, D. (2018). Cambios en la condición física en estudiantes que ingresan al programa de Cultura Física, Deporte y Recreación Cohorte 2013-I. Cuerpo, $\quad$ Cultura $y \quad$ Movimiento, $8(1), \quad 27-45$. https://doi.org/10.15332/2422474x/5120

Ceballos-Gurrola, O., Lomas-Acosta, R., Enríquez-Martínez, M. A., Ramírez, E., Medina-Rodríguez, R. E., Enríquez-Reyna, M. C., \& Cocca, A. (2020). Impact of a health program on metabolic profile and self-concept in adolescents with obesity. Retos, 83, 452-458.

Chamorro, R., Farías, R., \& Peirano, P. (2018). Circadian rhythms, eating patterns, and sleep: A focus on obesity. Revista Chilena de Nutricion, 45(3), 285-292. https://doi.org/10.4067/s0717-75182018000400285

Chavarría Sepúlveda, P., Barrón Pavón, V., \& Rodríguez Fernández, A. (2017). Nutritional status of active older adults and its relationship with some sociodemographic factors. Revista Cubana de Salud Publica, 43(3), 1-12.

Costa Acosta, J., Valdés López Portilla, M. R., Rodríguez Madera, A., Núñez González, A., Costa Acosta, J., Valdés López Portilla, M. R., Rodríguez Madera, A., \& Núñez González, A. (2021). PODIUM - Revista de Ciencia y Tecnología en la Cultura Física. Podium. Revista de Ciencia y Tecnología En La Cultura Física, 16(2), 369381.

Cruz E, Pont D, Cardoso C, Arredondo V, Gutiérrez M, Mendoza C, Obregón D, Sandoval A, Rojas B, Rosas L, \& Volantín F. (2020). Artículos de revisión Nutritional strategies in the management of patients with diabetes mellitus. Rev Med Inst Mex Seguro Soc, 58(1), 50-60.

Diaz, K., \& Development, A. C. (2021). arterial como factor de riesgo metabólico en estudiantes universitarios Asociación del índice de masa corporal y relación cintura / estatura con la presión arterial como factor de riesgo metabólico en estudiantes universitarios. December. https://doi.org/10.37527/2021.71.3.002

Dopsaj, M., Pajic, Z., Kocic, A., Erak, M., Pajkic, A., Vicentijevic, A., Milosevic, M., \& Bozovic, B. (2021). Profile for Body Fat Percentage of Serbian Working Population, 
Aged from 18 to 65, Measured by Multichannel Bioimpedance Method Perfil del Porcentaje de Grasa Corporal de la Población Activa Serbia, de 18 a 65 Años, Medido por el Método de Bioimpedancia M. Int. J. Morphol, 39(6), 1694-1700.

Espinoza-Navarro, O., Brito-Hernández, L., \& Lagos-Olivos, C. (2020). Composición Corporal y Factores de Riesgo Metabólico en Profesores de Enseñanza Básica de Colegios de Chile. International Journal of Morphology, 38(1), 120-125. https://doi.org/10.4067/s0717-95022020000100120

Fuentes-Barria, H., Aguilera-Eguía, R., \& González-Wong, C. (2021). Limiting factors of the physical training load in the covid-19 pandemic. Andes Pediatrica, 92(4), 641-642. https://doi.org/10.32641/ANDESPEDIATR.V92I4.3801

Hernández Rodríguez, José, \& Domínguez, A. Y. (2019). Principales elementos para tener en cuenta para el correcto diagnóstico de la sarcopenia. Medisur: Revista de Ciencias Médicas de Cienfuegos, 17(1), 112-125.

Hernández Rodríguez, Jose, \& Licea-Puig, M. E. (2017). Generalities and treatment of Sarcopenia. Revista de Los Estudiantes de Medicina de La Universidad Industrial de Santander, 2, 71-81.

Huaman, L., \& Bolaños, N. (2020). Sobrepeso, obesidad y actividad física en estudiantes de enfermería pregrado de una universidad privada. Enfermería Nefrológica, 23(2), 184-190.

López, M. N., Grassiolli, S., de Lá Ó Ramalho Veríssimo, M., de Oliveira Toso, B. R. G., Favil, P. T., de Paula, A. C. R., \& Viera, C. S. (2020). Dietary habits, anthropometric and metabolic profile of adolescents born prematurely. Journal of Human Growth and Development, 30(2), 241-250. https://doi.org/10.7322/JHGD.V30.10370

Mahecha, S. (2021). Poder del músculo esquelético en la salud y enfermedad. Rev. Nutr. Clin. Metab, 4(4), 4-18.

Mantilla-Morrón, M., Tepox-Bruno, R., Urina-Triana, M., Urina-Jassir, D., RebolledoCobos, R., Galeano-Muñoz, L., \& Gómez, L. (2018). Evaluación de la eficacia del ejercicio físico sobre la capacidad funcional de sujetos con enfermedad cardiovascular. Latinoamericana de Hipertensión, 13(6).

Massaroli, L. C., Santos, L. C., Carvalho, G. G., \& Carneiro, S. A. J. F. (2021). Qualidade De Vida E O Imc Alto Como Fator De Risco Para Doenças Cardiovasculares: Revisão Sistemática. Educação Física Para Grupos Especiais: Exercício Físico Como Terapia Alternativa Para Doenças Crônicas, 16, 122-132. https://doi.org/10.37885/210605072 
Navarrete, F. C., Hormazábal, M. A., \& Floody, P. D. (2015). Niveles de obesidad, perfil metabólico, consumo de tabaco y presión arterial en jóvenes sedentarios. Nutricion Hospitalaria, 32(5), 2000-2006. https://doi.org/10.3305/nh.2015.32.5.9619

Pedreros L, A., Calderón J, R., \& Moraga C, F. (2018). Estado nutricional, composición corporal e indicadores antropométricos de trabajadores mineros expuestos a hipoxia hipobárica crónica e intermitente a una altitud moderada (0-2500 msnm). Revista Chilena de Nutrición, 45(3), 199-204. https://doi.org/10.4067/s071775182018000400199

Pérez-López Eva, V.-A. A., \& Cambero-Rivero Santiago. (2020). Educación a distancia en tiempos de COVID-19: Análisis desde la perspectiva de los estudiantes universitarios [Distance education intimes of COVID-19: Analysis from the perspective of university students]. RIED. Revista Iberoamericana de Educación a Distancia, 24(1), 331-342.

Pérez Soto, J. J., García Cantó, E., Guillamón, A. R., Rodríguez García, P. L., Moral García, J. E., \& López García, S. (2019). Relación entre la intención de ser activo y la actividad física extraescolar. Revista de Psicología, 37(2), 389-405. https://doi.org/10.18800/10.18800/psico.201902.001

Silva Piñeiro, R. (2018). Los proyectos de caminata rumbo a la escuela para el conocimiento del entorno y favorecer actitudes y hábitos saludables desde educación infantil. Educación, 27(53), 177-202. https://doi.org/10.18800/educacion.201802.010

Tapia-Serrano, M. A., Pulido, J. J., Vaquero-Solís, M., Cerro-Herrero, D., \& SánchezMiguel, P. A. (2020). Systematic review about the effectiveness of physical activity programs to reduce overweight and obesity in school-age youth. Revista de Psicologia Del Deporte, 29(2), 83-91.

Velasco Estrada, A. S., Orozco González, C. N., \& Zúñiga Torres, M. G. (2018). Asociación de calidad de dieta y obesidad. Población y Salud En Mesoamérica, 1(1). https://doi.org/10.15517/psm.v1i1.32285

Villaquiran-Hurtado, A. F., Ramos, O. A., Jácome, S. J., \& Meza Cabrera, M. del M. (2020). Actividad física y ejercicio en tiempos de COVID-19. CES Medicina, 34, 51-58. https://doi.org/10.21615/cesmedicina.34.covid-19.6

Villena Chávez, J. E. (2018). Prevalencia de sobrepeso y obesidad en el Perú. Revista Peruana de Ginecología y Obstetricia, 63(4), 593-598. https://doi.org/10.31403/rpgo.v63i2034 
El artículo que se publica es de exclusiva responsabilidad de los autores y no necesariamente reflejan el pensamiento de la Revista Conciencia Digital.

\section{Ciencia
Digital
$\varepsilon_{\text {edtaol }}$}

El artículo queda en propiedad de la revista y, por tanto, su publicación parcial y/o total en otro medio tiene que ser autorizado por el director de la Revista Conciencia Digital.
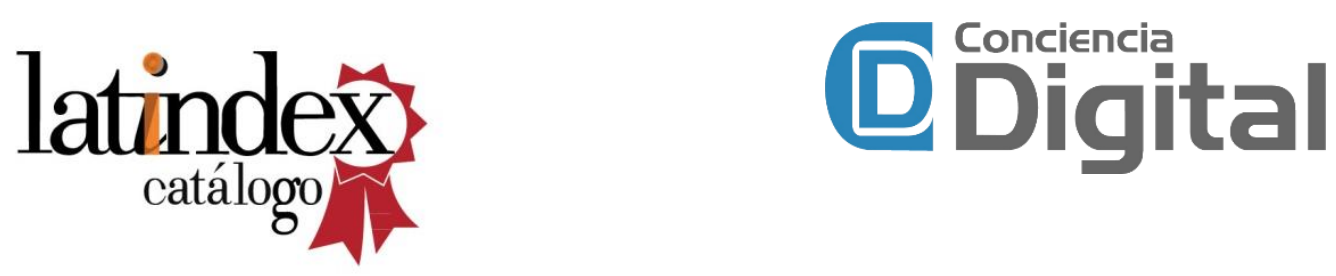

Indexaciones

doi crossref latindex̂.

